\title{
Prospección arqueológica submarina en la zona entre el Uadi Gawassis y Marsa Alam
}

Myriam SECO ÁLVAREZ

Se presentan los resultados de la primera intervención arqueológica submarina que tuvo lugar en la zona entre el Uadi Gawasis y Marsa Alam, en agosto de 2001, en colaboración con el Departamento de Arqueología Submarina del SCA, que consistió en su prospección visual en línea. Prestamos, entonces, especial atención a las bahías ligadas al interior a través de uadis, sobre todo aquellos que han servido de rutas para unir el Nilo con el Mar Rojo, como el Uadi Hammamat y el Uadi Gawasis.

La técnica utilizada fue la de prospección visual, lo cual nos permitió prospectar la zona de terrazas asociadas a la barrera de corales a la entrada de las bahías. Estas terrazas se sitúan generalmente a una profundidad entre $12-20 \mathrm{~m}$ y $50 \mathrm{~m}$. Ésta es la zona de mayor potencial arqueológico.

\section{Archaeological underwater survey in the area between Wadi Gawassis and Marsa Alam}

We present the results from the first underwater archaeological mission in the Red Sea, in the area between Wadi Gawassis and Marsa Alam, undertaken in August 2001, in collaboration with the underwater archaeological department from SCA. The work consisted of a visual survey on line. We concentrated our investigation on those bays linked to the interior by means of the wadis particularly those which served as routes connecting the Nile with the Red Sea, such as Wadi Gawasis and Wadi Hammamat.

We visually surveyed the areas at the entrance of those bays associated with coral reefs and underwater terraces at a depth from 10-20 $\mathrm{m}$ to $50 \mathrm{~m}$ : the depth with the highest archaeological potential.

KEY wORDs: Wadi Hammammat, wadi Gawasis, archaeological underwater survey

$\mathrm{L}$ a prospección arqueológica submarina en la zona entre el Uadi Gawassis y Marsa Alam, en colaboración con el Departamento de Arqueología Submarina del Consejo Supremo de Antigüedades Egipcias (SCA), tuvo lugar en el mes de agosto del año 2001. Dicha intervención ha estado precedida por una primera semana de prospección terrestre entre el uadi Gawasis y Marsa Alam, con el objeto de determinar los lugares que pueden resultar interesantes desde una perspectiva de arqueología subacuática a lo largo de nuestra concesión, lo que permitiría plantear intervenciones posteriores.

La elección del litoral del Mar Rojo para llevar a cabo la prospección arqueológica ha estado motivada por tres razones: históricas, arqueológicas y de protección de un patrimonio en peligro.

Desde un punto de vista histórico, el Mar Rojo ha tenido una gran importancia desde época fa- 
raónica, griega y romana, debido sobre todo a los intercambios comerciales con los países del Oriente Próximo, India, Extremo Oriente, el reino de Punt (fig. 1). La zona prospectada corresponde a la región más apta como punto de salida para estos destinos, pues es la zona del litoral que se encuentra más próxima al Valle del Nilo (fig. 2).

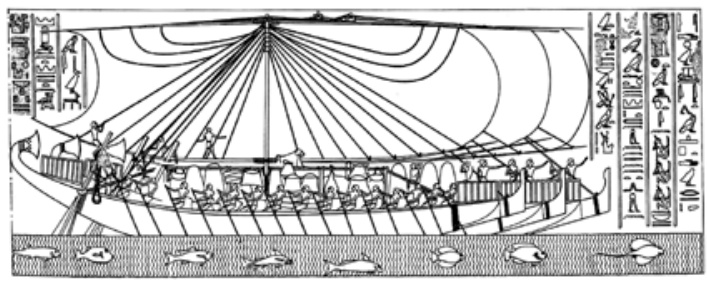

Figura 1

Desde un punto de vista arqueológico, se observa, por un lado, el aumento de excavaciones arqueológicas a lo largo de la costa del Mar Rojo y, por otro, la intensificación de estudios sobre rutas, fortificaciones, canteras y minas a lo largo del desierto oriental egipcio. Todo esto permite poner en relación yacimientos portuarios del Valle del Nilo con toda esta área. Por tanto, una prospección sistemática de la costa permitirá completar estos conocimientos. En el estado actual de éstos, las rutas comerciales partían del valle, atravesaban el desierto oriental y, finalmente, desembocaban en el litoral donde los productos se embarcaban.

Por último, la tercera razón que nos ha motivado a elegir esta zona es la protección de un patrimonio arqueológico en peligro que se encuentra amenazado debido a la gran expansión turística que experimenta la región.

Aunque se conozcan algunos pecios como el de Saadana, Quseir, Fury Shoal, y algunos restos de ánforas, aún no se tiene una idea objetiva de

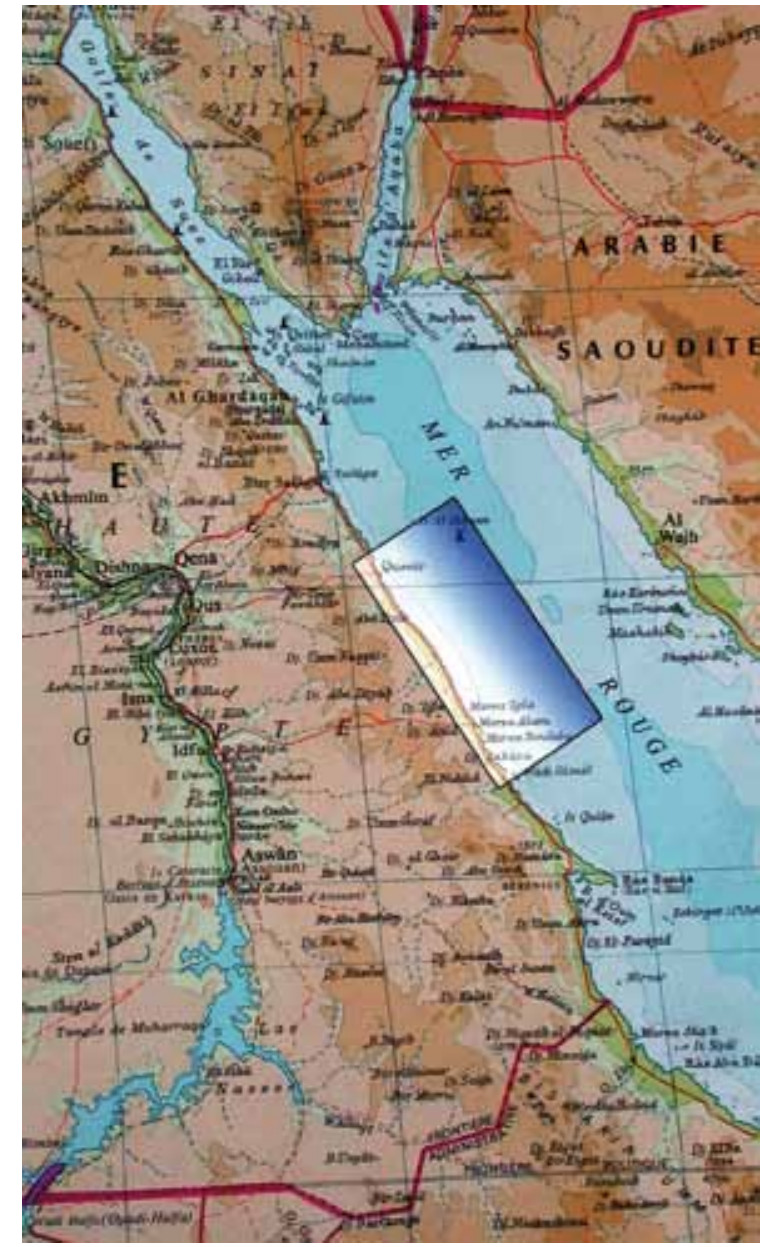

Figura 2

la riqueza de estos fondos. En realidad, sólo una prospección arqueológica submarina exhaustiva podrá completar estas lagunas.

A continuación presentamos los lugares prospectados durante esta primera misión, así como los descubrimientos realizados, en espera de que nuestras pistas de investigación y la metodología desarrollada sirvan para llevar a buen fin nuestros trabajos arqueológicos submarinos.

Comenzar nuestra prospección por el sur de la concesión, de Marsa El Naba El Sagheir a Marsa Nakari, nos ha permitido intervenir en una zona aún 
en expansión, en la cual la mayor parte de las estructuras de acogida turística se encuentran en construcción. Por tanto, es una zona virgen, recientemente desmilitarizada y en la que los restos arqueológicos no han sido aún descubiertos ni expoliados.

Desde un punto de vista histórico sólo el puerto romano de Nechesia, que se corresponde con Marsa Nakari, era ya conocido en todo este litoral de 50 $\mathrm{km}$. Por tanto, esta costa ha debido de tener circulación de embarcaciones que se dirigían hacia el sur del Mar Rojo, hacia el puerto de Berenice (fig. 3), la costa de Somalia, a Bab El-Mandeb y más lejos aún hacia el Océano Índico.

La realización de una prospección arqueológica submarina en el Mar Rojo es un proyecto inédito $\mathrm{y}$, por tanto, los resultados no pueden evaluarse fácilmente. Es necesario establecer un método adaptado a la realidad de este litoral. La finalidad de esta primera misión, de un proyecto que durará varios años, ha sido evaluar las condiciones que presenta la costa con el fin de alcanzar unos primeros objetivos que permita estudiar, en el futuro, los fondos de este espacio de manera rigurosa:

1. La prospección sistemática de todo el litoral incluido en la concesión. El estudio de los fondos submarinos ha estado apoyado por un análisis de la zona terrestre limítrofe. Por una parte, hay que tener en cuenta la gran profundidad de la placa costera interior a escasa distancia de la orilla y, por otra

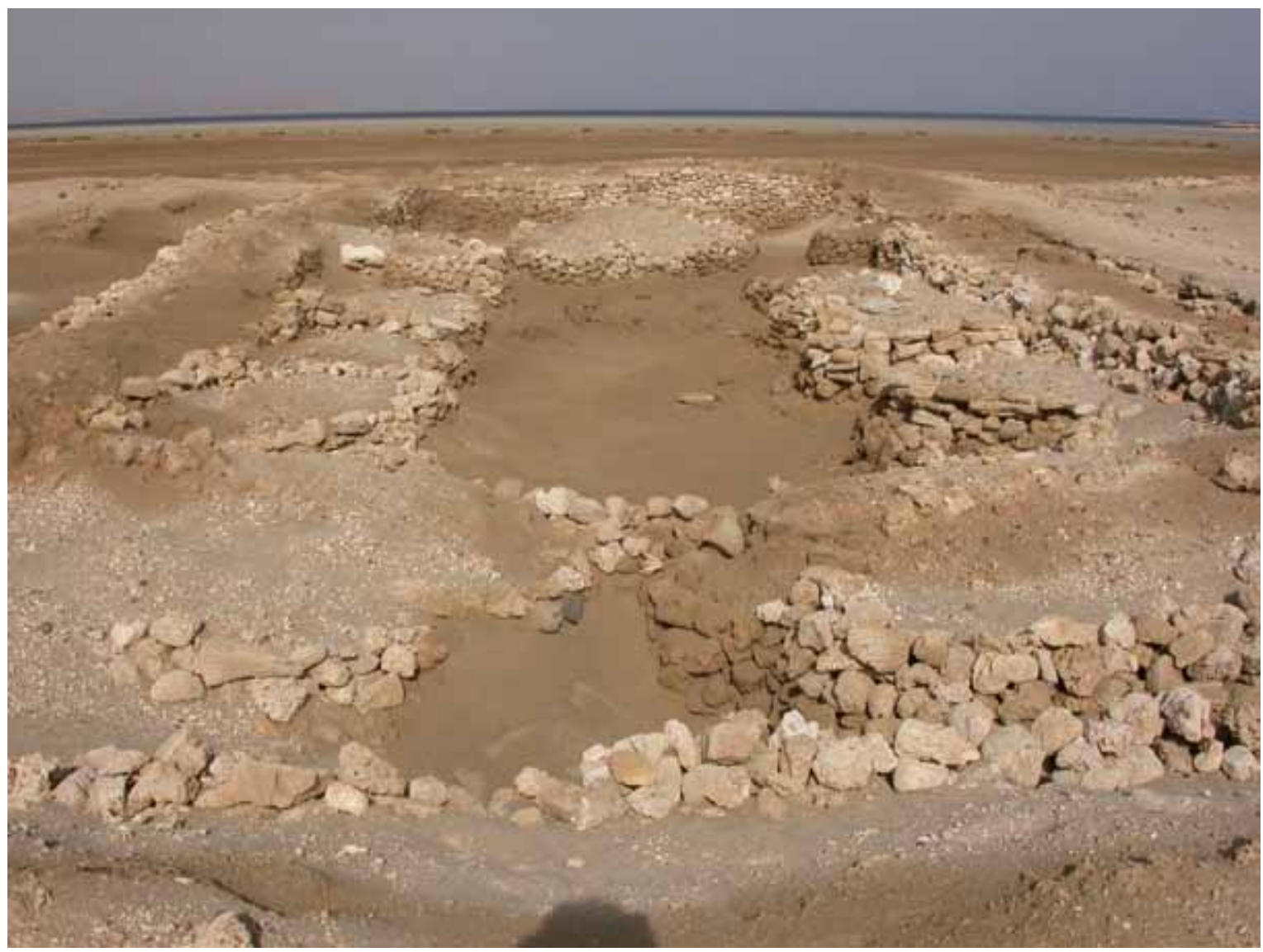


parte, que el tipo de navegación realizado era de cabotaje diurno. En realidad, hay que señalar que la costa del Mar Rojo ofrece muy pocas bahías resguardadas que hayan podido ser utilizadas por las naves antiguas. Tan solo el 9\% del litoral puede servir geomorfológicamente como resguardo para las embarcaciones. El resto son paredes que en algunos casos bajan a 40 o $50 \mathrm{~m}$ y en otros sobrepasan los 100. Por este motivo las inmersiones han estado precedidas por una prospección terrestre. Ésta nos ha servido para definir las zonas que pueden ser propicias para el anclaje o refugio de navíos y para elegir las zonas en las que realizar las prospecciones submarinas.

2. El inventario de los vestigios submarinos. Esto permitirá identificar y registrar el patrimonio existente.

3 . Proteger y conservar los vestigios arqueológicos sumergidos en zonas como ésta, donde hay una gran actividad deportiva de buceo. Se pretende sensibilizar los centros de buceo y dar una identidad histórica a este litoral en vías de expansión turística.

4. Elaboración de una carta arqueológica submarina, lo cual permitirá evaluar el patrimonio existente, así como estudiar y comparar los diferentes tipos de vestigios y definir las zonas principalmente utilizadas como anclaje o los trayectos de circulación [236] dominantes.

El litoral en esta zona está orientado de noreste a suroeste, con vientos del noroeste y corrientes del norte con la marea baja y del sur con la marea alta. Las condiciones climáticas hacen de este mar una zona de navegación difícil. En toda esta costa desemboca una serie de ríos actualmente secos, formados en la época del pleistoceno a partir de lluvias torrenciales. Estos ríos han sido utilizados como vías de circulación preferida por los antiguos y, por otro lado, ocasionaron la formación de bahías en la zona costera y manglares (fig. 4).

La infraestructura de la línea de costa está compuesta por marsa, sharm y halk.

Los marsa son bahías alargadas en la desembocadura de un río. Normalmente tienen un fondo de arena, una pequeña playa y barrera de corales al nivel de la apertura hacia el mar. Al estar protegidos de las corrientes y de las mareas, presentan una zona resguardada e idónea para el anclaje. El término árabe "marsa" designa una bahía natural situada en la desembocadura de un uadi y formada por el flujo de las aguas torrenciales de lluvias a través de este uadi. Este flujo transforma los sedimentos en un fondo arenoso que permite subir las naves hasta la ribera. Dependiendo del tamaño y del relieve del marsa y de la barrera de corales que tiene asociada, los barcos que allí se refugian estarán más o menos protegidos de las corrientes $\mathrm{y}$ los vientos.

Los sharm son pequeñas bahías en forma de V, más pequeños que los anteriores y menos protegidos contra las corrientes y los vientos. También podían servir de resguardo para pequeñas embarcaciones. El término árabe originariamente se corresponde con el labio inferior del camello, que hace pensar en una $\mathrm{V}$, y de ahí, por analogía, estas bahías toman su nombre.

Por último, los halk son bahías cerradas por la barrera de corales. Son muy peligrosos para la navegación, porque son parecidos a los marsa pero no son utilizables como resguardo.

Teniendo en cuenta estos factores, nuestras prospecciones submarinas se dirigen principalmente a los marsa (tanto a su zona terrestre como a la subacuática), pero también a algunas barreras de corales prominentes en toda su longitud. 


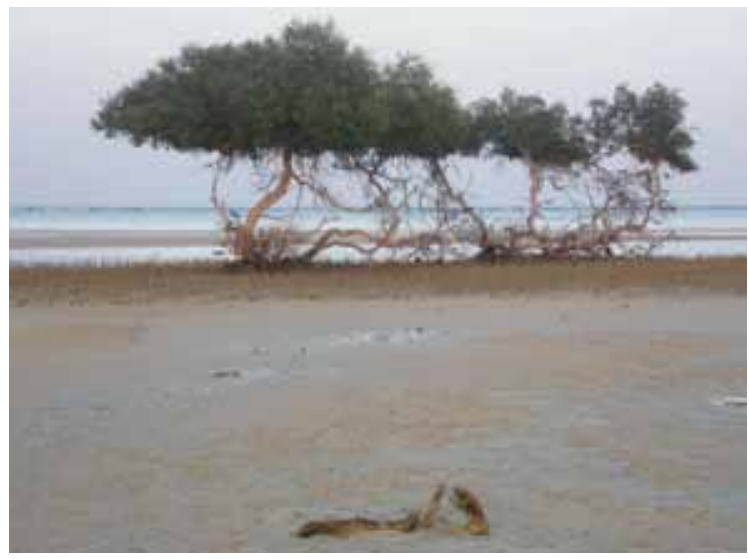

Figura 4

\section{Prospecciones terrestres}

Las prospecciones se han efectuado a lo largo del litoral sobre una banda de $500 \mathrm{~m}$ de anchura. Hemos prestado especial atención a las bahías ligadas al interior a través de uadis, sobre todo aquellos que han servido de rutas para unir el Nilo al Mar Rojo.

La utilización de un GPS manual nos ha permitido localizar los yacimientos y encontrar bahías importantes que conocíamos a través de nuestros mapas. Para llevar a cabo este trabajo hemos utilizado los planos del Egyptian General Survey Authority (Egyptian Series 1/50 000) ya que su escala es adecuada y nos ha permitido leer las variaciones en la línea de costa. En realidad, no existe aún una carta marina y batimétrica precisa. Los puntos GPS han sido descargados en un ordenador portátil a través del programa Fugawi 3, que permite georeferenciar los mapas: cada punto medido es automáticamente incluido en el total de la zona.

Marsa uadi Gawassis

(2634'20,90”'N / 3402'07,90”'E)

Este puerto se sitúa a $24 \mathrm{~km}$ al sur de Safaga. Existen restos de anclas de piedra caliza dispersos por diferentes lugares. En el interior del uadi hemos podido observar gran cantidad de cerámica procedente de excavaciones llevadas a cabo en la década de $1970^{1}$. Las anclas de piedra caliza se encuentran en muy mal estado de conservación. También existe aún una estela de granito con restos de inscripciones jeroglíficas, pero totalmente deteriorada. Teniendo en cuenta todos estos vestigios hemos llevado a cabo varias inmersiones submarinas en la zona.

Marsa uadi Gassous

(2634'25,1'”N/3401'47,6”'E)

Bahía situada a $2 \mathrm{~km}$ al norte de la precedente y formada por un uadi de mayores dimensiones que el anterior. Actualmente el puerto Gassous está ocupado por un centro de buceo deportivo, The Coral Garden Resort. En las prospecciones que allí hemos realizado han aparecido algunos fragmentos de cerámica. Los integrantes del centro nos han señalado que en el extremo noroeste de la bahía existe un ancla de hierro de sección cuadrada a una profundidad de $40 \mathrm{~m}$.

\section{Myos Hormos

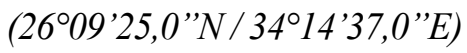

El antiguo puerto de Myos Hormos está situado en Quseyr El-Qadim². La Universidad de Southampton lleva a cabo en él excavaciones sistemáticas. Hay que señalar la existencia de un pecio romano con una importante carga de ánforas, localizado al norte de esta bahía a unos $64 \mathrm{~m}$ de profundidad. Debido a la cota en que se encuentra no ha sido expoliado.

Mangrove Bay (Marsa Fugani) (2552'06,2”N / $\left.34^{\circ} 24^{\prime} 50,2^{\prime \prime} \mathrm{E}\right)$

La forma de Marsa Fugani, actualmente ocupada por unas instalaciones turísticas, es ideal para proteger varios navíos. Esta bahía presenta 
una longitud fuera de lo corriente y dos promontorios bastante elevados al sur y al norte que sirven para cortar el viento. La barrera de corales ofrece una protección contra las corrientes y la entrada al marsa está dominada por una playa arenosa que servía de acceso a las embarcaciones. Se observan también restos de cerámica en la misma playa. Los buceadores de Mongrove Bay nos han hablado de la presencia de tres anclas de hierro situadas a lo largo de la barrera de corales, hacia el norte de este enclave.

\section{Marsa Gabel El-Roussas}

(2552'14,4'” $N / 34^{\circ} 25^{\prime} 07,3$ '” $\left.E\right)$

Se trata de una bahía muy cerrada y, por tanto, sirve de resguardo ideal a las embarcaciones. Tiene una pequeña playa arenosa y está protegida por dos promontorios, tanto al norte como al sur, bastante elevados. Hay que tener en cuenta la importancia de los fuertes vientos en esta costa del Mar Rojo. Hemos hallado una rueda de molino en granito de Asuán de $114 \mathrm{~cm}$ de diámetro por $50 \mathrm{~cm}$ de altura (Fig.5). Está seccionada en dos y se encuentra a unos metros del agua. Hemos decidido llevar a cabo varias inmersiones en este lugar.

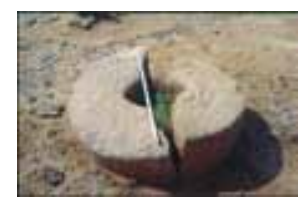

Marsa Nakari

Figura 5 $\left(24^{\circ} 55^{\prime} 36,1^{\prime \prime} N / 34^{\circ} 57^{\prime} 41,1^{\prime \prime} E\right)$

[ 238 ] Esta bahía es bastante pequeña y bien protegida; en la actualidad sirve de resguardo a las embarcaciones. Tiene una pequeña playa arenosa y tanto al norte como al sur está resguardada por unos promontorios. La barrera de corales es muy prominente al sur, y alcanza una longitud que supera los $100 \mathrm{~m}$. En el promontorio meridional, un yacimiento, cuya excavación se ha iniciado re- cientemente, se ha identificado con la antigua Nichesia (Fig. 6). Teniendo en cuenta estos vestigios arqueológicos terrestres, efectuar varias intervenciones submarinas resulta de gran interés.

\section{Prospecciones submarinas}

La técnica utilizada en esta primera campaña ha consistido en la prospección visual en línea. El equipo de arqueólogos submarinos se disponía en forma perpendicular a la barrera de corales. Este método permite prospectar visualmente la zona de terrazas asociadas a la barrera coralina a la entrada de las bahías. Estas terrazas se sitúan generalmente a una profundidad de entre $12-20 \mathrm{~m}$ hasta $50 \mathrm{~m}$. Es en esta zona donde se pueden encontrar los vestigios de pecios y es la única accesible a los buceadores con aire comprimido. Sin embargo, las prospecciones realizadas en el interior de la bahía las hemos hecho en dos grupos de dos arqueólogos con el objeto de cubrir el mayor espacio posible. Normalmente nos hemos dejado llevar por la corriente. Algunos de los yacimientos no son accesibles desde la costa y por tanto ha sido necesaria la utilización de una pequeña embarcación.

Bahia de Marsa Gawassis

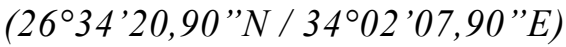

Es la situada más al norte en nuestras prospecciones. Su fondo presenta gran cantidad de arena, lo cual obstaculiza la localización de restos antiguos salvo que se disponga de material de trabajo adecuado. En la próxima campaña se intervendrá en esta zona con un sonnar lateral o sub-bottom-profiler. 


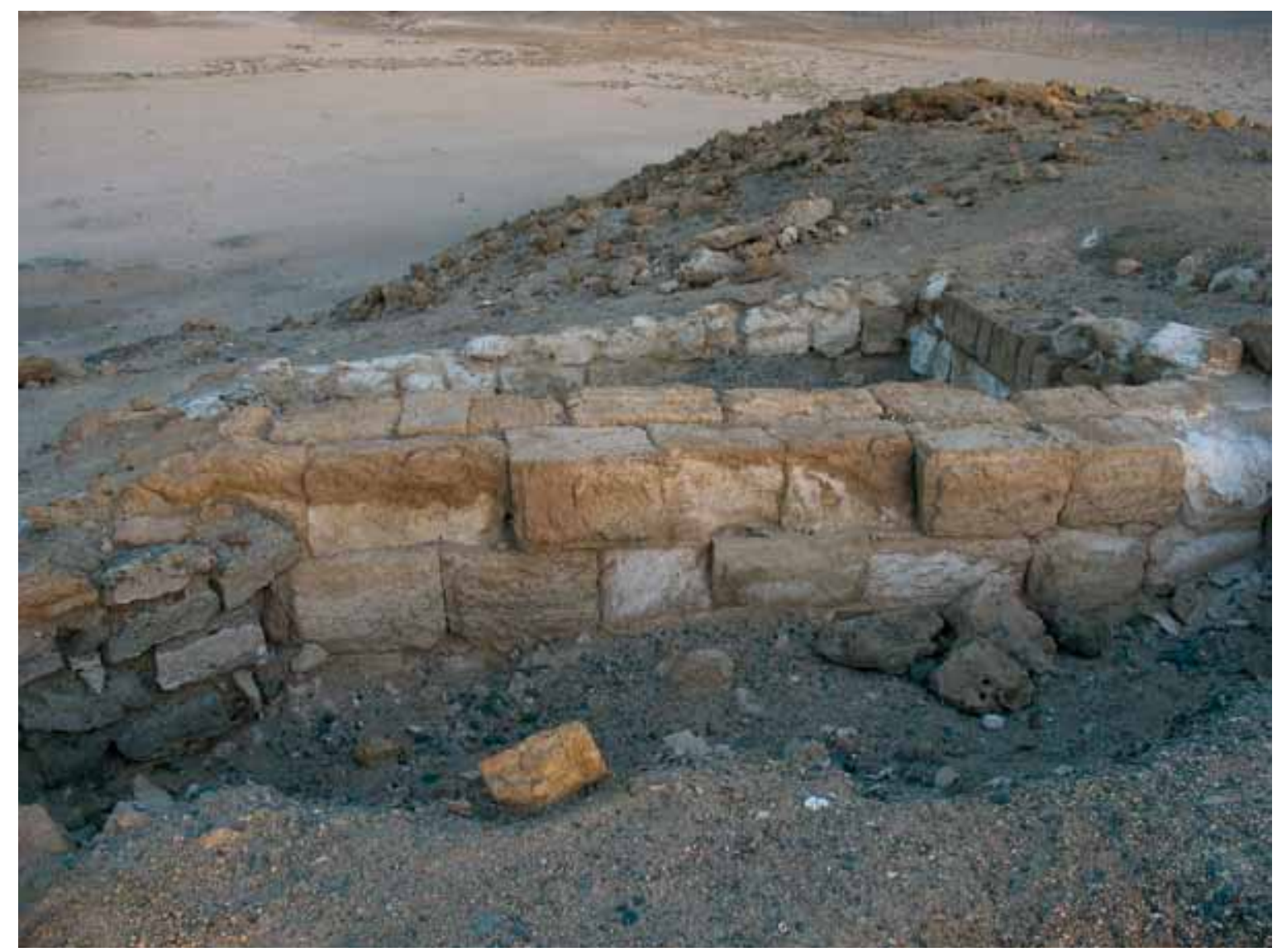

Figura 6

Bahía de Marsa Shagara

(2514'43,02'” N / 3447'35,91'”E)

Es de tamaño pequeño y presenta una barrera de corales al norte bastante prominente, lo cual la protege de las corrientes. Hemos encontrado un ancla de hierro situada al sur de la marsa a $32 \mathrm{~m}$ de profundidad que parece ser del S. XVIII; además, un ánfora romana, situada a 27 m en el medio de la bahía y otra árabe.

\section{Marsa Gabel El-Roussas}

$\left(25^{\circ} 12^{\prime} 16,05^{\prime \prime} \mathrm{N} / 34^{\circ} 48^{\prime} 24,21^{\prime \prime} \mathrm{E}\right)$

Se encuentra especialmente protegida y, por tanto, es un lugar ideal para el anclaje de pequeñas embarcaciones. Como ya se ha señalado, hemos encontrado una piedra de molino de granito rojo.
Las primeras jornadas hemos trabajado a partir de la costa. Se han cubierto las barreras de corales norte y sur. Durante los siguientes días ha sido necesaria la utilización de una pequeña embarcación con el fin de cubrir el exterior de la bahía. Siguiendo la técnica de prospección visual en línea, no se ha observado encontrado ningún vestigio. El fondo del marsa es bastante arenoso, lo que la convierte en un lugar ideal para prospectar con el sonnar.

\section{Marsa uadi Assila \\ (2509'16,37'” N / 34'51'06,81 '”E)}

Bastante protegida contra vientos y mareas al tener una barrera de corales muy prominente al norte. Las prospecciones se han llevado a cabo a partir de una embarcación y 
hemos cubierto tanto la zona norte como la sur. Ningún vestigio relevante ha sido identificado.

\section{Bahía de Marsa Nakari}

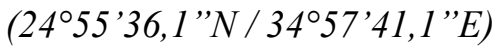

Como ya lo hemos presentado, el promontorio meridional se esta excavando. Bahía bastante protegida contra los vientos y las mareas. Por otro lado, presenta una barrera de corales al sur bastante prominente que puede haber sido la causa de accidentes de navegación. Esta barrera supone un espacio idóneo para prospectar. La bahía presenta un fondo arenoso que dificulta la identificación de restos arqueológicos. Es un lugar apropiado para utilizar otras técnicas de prospección como la del sonnar.

\section{Exterior del puerto de Marsa Alam}

Es un área muy extensa y han sido necesarias varias inmersiones para cubrirla por completo. Los fondos están poco arenados y la terraza es muy extensa. Hemos encontrado tres ánforas romanas a una profundidad de $16 \mathrm{~m}$. incrustadas en el coral. Es una zona que necesita nuevas inmersiones para poder evaluar los restos arqueológicos.

Se constata que en próximas intervenciones será necesaria la utilización de material submarino de succión de sedimento, para poder efectuar sondeos en zonas como el uadi Gawasis, uadi Hammamat o marsa Nakari, donde existen claros restos arqueológicos en tierra. Por otro lado, será necesario un material de prospección magnético adecuado para detectar vestigios en estos lugares, así como necesario para la elaboración de una carta arqueológica precisa. 


\section{Bibliografía}

SAYED, A.-M.

1979 Discovery of the Site of the 12th Dynasty Port at Wadi Gawasis on the Red Sea Shore, Actes First International Congress of Egyptology. Cairo, October 2 - 10, 1976, Berlin: 569-577.
Whitcomb, D.

1996 Quseir Al-Qadim and the location of Myos Hormos, Topoi 6 (2): 747-772. 


\section{Trabajos de Egiptología Papers on Ancient Egypt}

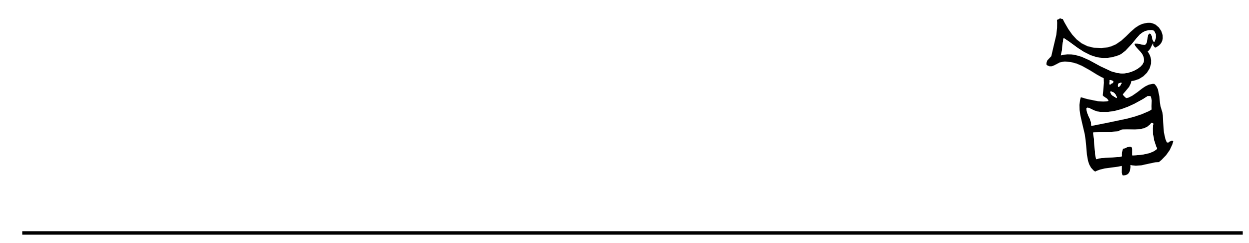

Número 5/2 2009 


\section{Actas \\ III Congreso Ibérico de Egiptología III Congresso Ibérico de Egiptologia}

Editores

Miguel Ángel Molinero Polo Covadonga Sevilla Cueva 


\title{
Editor
}

Miguel Ángel Molinero Polo

Universidad de La Laguna

\section{Consejo Editorial}

\author{
Antonio Pérez Largacha \\ Universidad de Castilla-La Mancha \\ José-R. Pérez-Accino \\ Birkbeck, Universidad de Londres \\ Covadonga Sevilla Cueva \\ Universidad Autónoma de Madrid
}

\section{Comité Científico}

Josep Cervelló i Autuori

Universitat Autònoma de Barcelona

$\mathrm{M}^{\mathrm{a}}$ José lópez Grande

Universidad Autónoma de Madrid

Josep Padró i Parcerisa

Universitat de Barcelona

$\mathrm{M}^{\mathrm{a}}$ Carmen Pérez Die

Museo Arqueológico Nacional, Madrid

Ester Pons Mellado

Museo Arqueológico Nacional, Madrid

José M. Serrano Delgado

Universidad de Sevilla

\section{Colaboradores Editoriales}

Linda Steynor

English editorial assistant

Hervé Mouriacoux

Assistant éditorial pour la langue française 
Trabajos de Egiptología está producida por Isfet. Egiptología e Historia c/ Blanco $1,2^{\circ}$

38400 Puerto de la Cruz

Tenerife-Islas Canarias

España

Maquetación: Proyecto Limón

(C) Autores de los artículos aparecidos

y Consejo Editorial de Trabajos de Egiptología - Papers on ancient Egypt

Depósito Legal: TF-2303-2009

ISSN: $1695-4750$

Imprime: Gráfica Los Majuelos, S.L.L.

imprenta@graficaslosmajuelos.com

Tfno.: 922311455 


\section{Comité Científico \\ III Congreso Ibérico de Egiptología III Congresso Ibérico de Egiptologia}

Miguel Á. Molinero Polo

Universidad de La Laguna

Presidente del Comité Organizador del III Congreso Ibérico de Egiptología

Miembro del Comité Organizador del I Encuentro de Egiptología

Josep Cervelló Autuori

Universitat Autònoma de Barcelona

Presidente del Comité Organizador del II Congreso Ibérico de Egiptologia

José Manuel Galán Allué

Consejo Superior de Investigaciones Cientificas

Director del Proyecto Djehuty, Luxor, Egipto

$\mathrm{M}^{\mathrm{a}}$ Helena Trindade Lopes

Universidad de Lisboa

Directora de la Misión Arqueológica Portuguesa en Menfis

Josep Padró i Parcerisa

Universitat de Barcelona

Director de la Misión Arqueológica de Oxirrinco

Antonio Pérez Largacha

Universidad de Castilla - La Mancha

Miembro del Comité Organizador del I Encuentro de Egiptología

José Ramón Pérez-Accino

Birkbeck College, University of London

Miembro del Comité Organizador del I Encuentro de Egiptología

$\mathrm{M}^{\mathrm{a}}$. Carmen Pérez Díe

Museo Arqueológico Nacional

Directora de la Misión Arqueológica Española en Heracleópolis Magna, Egipto

Covadonga Sevilla Cueva

Universidad Autónoma de Madrid

Miembro del Comité Organizador del I Encuentro de Egiptología 